\title{
Graduate Student Difficulties on Electrostatics Topics -- Conductors and Insulators
}

\author{
Qiaoyi Liu ${ }^{1, *}$ \\ ${ }^{1}$ Department of Physics, The Ohio State University, Columbus, Ohio 43210, USA \\ *Correspondence: liu.6530@osu.edu
}

(Received: 09/24/2019; Accepted: 11/10/2019; Published: 11/20/2019)

DOI: https://doi.org/10.37906/real.2019.1

\begin{abstract}
A lack of conceptual understanding of electrostatics principles, especially in the context of conductors and insulators, has been observed among a large number of introductory physics students as well as upper-level physics students. This work aims to explore graduate students' misconceptions on these topics, particularly on how charges are distributed on conductors and insulators in various scenarios. Four first-year physics graduate students were selected to participate in think aloud interviews, where they were given a set of questions related to basic concepts of conductors and insulators, and asked to explain their thought processes as detailed as possible. The result of the four interviews are categorized by the test questions, which discusses the misconceptions that were demonstrated throughout the interviews, along with the interviewees' reasoning behind these misconceptions. Despite that the interviewees have taken the introductory and advanced electricity and magnetism courses, a number of misconceptions still prevail in their conceptual understanding related to conductors and insulators. One possible explanation is the discontinuity between their knowledge of mathematical formulation and physical understanding of the concept, thus it is recommended that instructors should enforce students to review the advanced theory in the context of physical situations.
\end{abstract}

Keywords: physics education; electricity and magnetism; graduate student

\section{Introduction}

Topics on conductors and insulators have been central to the electricity and magnetism (E\&M) curriculum, starting from the introductory sequence, where basic phenomenology is established, to the graduate level course, where more physical laws and mathematical tools (such as Laplace's equations, method of image charge, Green function, etc.) are taught. However, despite that such emphasis is put on these topics throughout the curriculum, numerous misconceptions still prevails among the students.

As a matter of fact, a limited number of studies have been conducted to probe students' understanding of these topics. A conceptual survey on general E\&M topics - the Conceptual Survey of Electricity and Magnetism (CSEM) - is widely used to probe students' misconceptions (Planinic, 2006; Demirci, 2005), yet it only contains four questions related to conductors and insulators, namely questions 1, 2, 13 and 14. Maloney et al. (2001) demonstrated that a large number of introductory physics students failed to answer these questions correctly both during pre- and post-testing. Their study had shown that many students 
may simply recall answers without understanding the physical mechanism, and that students' knowledge of the shielding effect of conductors seems rather weak.

Hazelton et al. (2013) produced a focused study that probed introductory physics students on their application of electrostatic laws to conductors. They demonstrated that throughout the entire course of instruction, students have significant difficulties in relating electrostatics concepts (e.g. electric potential, charge distribution) and connecting the mathematical formulation with the physical concept. However, the study did not address students' understanding of the electric field distribution over all space due to the charge distribution, which is an essential concept that is deeply connected with the charge distribution and can help explain students' misconceptions related to conductors and insulators.

This work aims to build on the previous studies, and use a problem set with questions that combines more contextual variables that relate to conductor and insulator concepts, as to further probe students' conceptual understanding on conductors and insulators.

\section{Research Design}

Four first-year physics graduate students at a U.S. research university were given a set of questions that were designed to probe basic concepts related to conductors and insulators. These students participate in think aloud interviews, where they were asked to explain their thought processes when solving these problems, in as much detail as possible (Chi, 1994). One incentive to choose this population is that graduate students' conceptual understanding has not been frequently studied, yet it has the potential to produce promising results, as difficulties on related concepts, e.g. Gauss's Law, have been identified among upperlevel undergraduate (Pepper, Chasteen, Pollock, \& Perkins, 2010).

The question set is designed explore various electrostatics concepts-such as charge distribution, electric field distribution, the charge neutrality of the object, etc. -in the context of conductors and insulators. By including a wide range of contextual variables, students' reasoning processes can be detailed as they demonstrate any logical (or illogical) connections made among them. The question set begins with a warm-up question which is intended to not only serves as a diagnostic tool, but also helps to refresh the interviewees' memory of relevant concepts that they may need for later problems. Next, students answer two multiple-choice questions regarding charge distribution on conductors and insulators, which are variations of problem 1 and 2 from CSEM. The original CSEM questions only considered positive charges inside the sphere; however, inclusion of both positive and negative adds an extra dimension that is crucial to describing the charge distribution, and a potential pitfall for conceptual understanding. Thus, in this question set, options are given for choosing positive or negative charge on the inside of the sphere. Finally, the set concludes with two show-work problems regarding charge induction on conductors, where the students have freedom to draw the charge distribution and electric field lines with minimal hints from the problem. The full problem set is included in the Appendix A.

\section{Analysis and Results}

This section discusses the result of the four interviews, categorized by the test questions. Each part starts with a brief description of the question that is being asked, what the desired answer is for that question, then discusses the difficulties that were demonstrated throughout the interviews, along with the interviewees' reasoning behind their responses.

\subsection{Question 1 - Warm-up Question}

The first question asks students to describe the fundamental properties of conductors and insulators.

All four interviewees mentioned various properties of conductors and insulators from different 
perspectives, which were all valid answers. The responses are summarized in Table 1. The most fundamental property of conductors and insulators that is relevant to the later problem is shown in the first row, i.e. whether or not the material allows charge to move freely. These responses demonstrate that the interviewees were able to recall fundamental rules (at least mechanically) related to conductors and insulators.

Table 1. Summary of student responses to question 1.

\begin{tabular}{cc}
\hline \hline Conductors are materials where... & Insulators are materials where... \\
\hline charges are free to move. & the motion of charges are not readily permitted. \\
electric field is zero inside, and all charge & charge is static, and does not allow flow of \\
resides on the surface. & electron, or passage of current. \\
resistance is low (zero). & resistance is high (infinite). \\
the valence band overlaps the conduction band. & $\begin{array}{c}\text { the valance band is separated by a large energy } \\
\text { gap from the conduction band. }\end{array}$ \\
\hline \hline
\end{tabular}

\subsection{Question 2 - Multiple-Choice Questions}

The second problem asks the students to identify the correct charge motion when an excess negative charge is placed on the hollow sphere. The case where the hollow sphere is a conductor is discussed in part (1), whereas the case where it is an insulator is in part (2).

For part (1) of the problem, in the metal sphere case, the correct answer is (b) the negative charge is evenly distributed over the outside of the sphere, while the inside of the sphere is electrically neutral.

However, 2 interviewees have chosen (c) the negative charge is evenly distributed both over the inside and outside of the sphere. One argued that "since negative charges repel each other, the excess charges will spread themselves evenly over both surfaces," while the other created the following scenario, and used "topological equivalence" to defend his idea: "If we consider a thick conducting sheet with the negative charge evenly distributed over both surfaces, we can take the limit of it being bent and wrapped into a spherical shell, so we should have the negative charge is evenly distributed both over the inside and outside of the sphere."

The other 2 interviewees have chosen (d) the negative charge is evenly distributed over the outside of the sphere, while positive charge is induced over the inside of the sphere. One ignored the fact that the conductor is charged: "since the conductor is neutral, and there is negative charge over the outside of the sphere, we need positive charge over the inside of the sphere to balance it out." The other claimed that "the negative charge over the outside of the sphere will induce a dipole moment across the conducting shell, thus create positive charge over the inside of the sphere."

After the interview, when all four interviewees were asked to check if their responses were consistent with Gauss's Law, and the fact that electric field is zero inside the conductor, they were all able to realize the discrepancy, and corrected their answers. This implies that while they may understand how to apply Gauss's Law in the context of a conductor, they lack the awareness to check their answers with it.

For part (2) of the problem, in the electrically insulating sphere case, the correct answer is (a) all of the negative charge remains right around the point $P$.

3 interviewees were able to obtain the correct answer, with the correct reasoning, while 1 picked (d) negative charge is evenly distributed over the outside of the sphere, while positive charge is induced over the inside of the sphere, with the same argument that a dipole moment is induced across the spherical shell. 


\subsection{Question 3 - Show-Work Problems}

The third problem focuses on the charge induction phenomenon for a conducting spherical shell, where a positive charge in placed inside the shell, and the students are asked to describe the charge distribution and electric field distribution. The case where the charge is placed at the center is discussed in part (1), whereas the case where the charge is off center is discussed in part (2).

A sample correct drawing of the charge and electric field distribution for part (1) and (2) of this problem is depicted in Figure 1, (a) and (b).

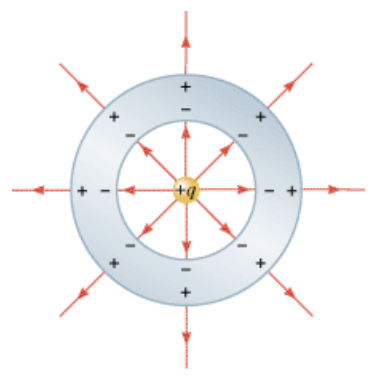

(a)

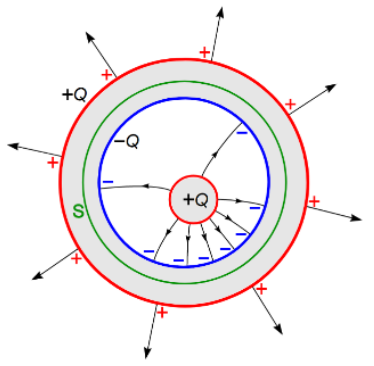

(b)

Figure 1. (a) Sample correct drawing for part (1); (b) Sample correct drawing for part (2).

For part (1) of the problem, the correct answer should show that the negative charge (-q) is induced and evenly distributed over the inside of the sphere, while positive charge $(+q)$ is induced and evenly distributed over the outside of the sphere, as shown in Figure 1 (a).

3 interviewees were able to produce the correct drawing utilizing the spherical symmetry of the setup, while 1 produced the drawing shown in Figure 2 (a) below, where the negative charge (-q) is induced and evenly distributed over the inside of the sphere, and the inside of the sphere is electrically neutral.

This student argued that "An observer from the outside should not be able to observe the charge due to electric shielding. The conductor serves as a Faraday's cage that shields the electric field from inside the cavity, which means that there should be no charge on the outside of the sphere." This reasoning further led this student to draw a similar charge distribution for part (2), (A).

After the interview, when this student was asked to check if his drawing was consistent with conservation of charge, and the fact that the conductor is electrically neutral, he was able to realize the discrepancy, and corrected his answer.

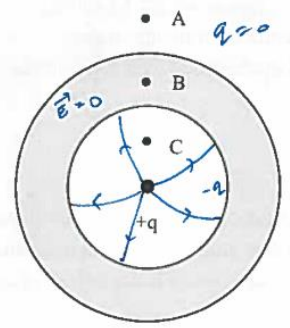

(a)

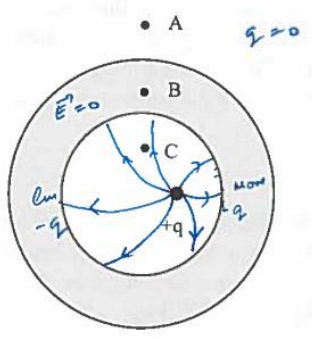

(b)

Figure 2. Sample student response for part (1) and (2). 
For part (2), (A) of the problem, the correct answer should show that over the inside of the sphere, more negative charge is induced near $+q$, less negative charge is induced away from $+q$, while over the outside of the sphere, positive charge $(+q)$ is induced and evenly distributed, as shown in Figure $1(b)$.

1 interviewee was able to produce the correct drawing, arguing that "since the electric field inside the conductor is zero, the inside of the sphere does not communicate with the outside." Two other student responses for this part are shown in Figure 3.

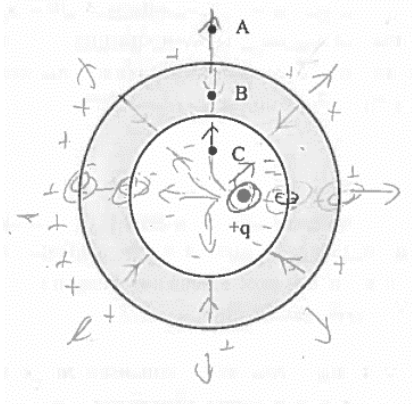

(a)

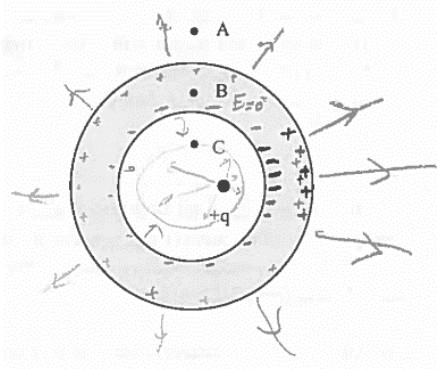

(b)

Figure 3. Additional sample student responses for part (2).

One response showed that over the outside of the sphere, less positive charge is induced near $+q$, and more positive charge is induced away from $+q$, as is shown in Figure 3 (a). This student argued that "since the negative charge over the inside of the sphere is more densely populated near $+q$, the effective charge near that region should be less positive." The other response showed that over the outside of the sphere, more positive charge is induced near $+q$, and less positive charge is induced away from $+q$, as is shown in Figure 3 (b). This student argued that "since the negative charge over the inside of the sphere is more densely populated near $+q$, while opposite charges attract."

For part (2), (B) of the problem, the correct answer is that the electric field is the same at point A and B, but different at point $C$.

1 interviewee was able to obtain the correct answer, with the correct reasoning. 2 interviewees claimed that the electric field is the different at point $\mathrm{A}$, which follows from their previous conclusions where the positive charge is asymmetrically distributed over the outside of the sphere. The last interviewee claimed that the electric field is the same at point $C$, arguing that "even though point $C$ is now far away from $+q$, the induced charge distribution over the inside of sphere has also changed, which will enforce the electric field to be the same at point C."

For part (2), (C) of the problem, the correct answer should show that inside the cavity, the electric field lines are denser over the region where $+q$ is closer to the inner surface, while outside the spherical shell, the electric field lines should resemble a point charge.

1 interviewee was able to produce the correct drawing, yet upon further questioning, he revealed that he simply recalled the answer from his memory with little justification. 1 interviewee believed that the electric field is non-zero inside the conductor, as is shown in Figure 3 (a), arguing that "the common property of the conductor that electric field is always zero inside the conductor does not apply here, since there is an excess charge that is inside the cavity, which will produce an electric field that will force the conductor to be at a non-equilibrium state at all time." 1 interviewee showed that inside the cavity, there exists a region where the electric field is zero, as is shown in Figure $3(b)$, saying that "in this region, the 
electric field from the point charge and that from the induced charge on the inside surface should cancel out." The same interviewee also showed that outside the spherical shell, the electric field lines are denser over the region where $+q$ is closer to the inner surface, which follows from his previous conclusion where the positive charge is asymmetrically distributed over the outside of the sphere.

\section{Conclusions and Recommendations}

Despite that the first-year physics graduate students interviewed had already taken the introductory and advanced electricity and magnetism courses, a number of misconceptions still prevail in their understanding of conductors and insulators. As can be seen in the responses of the interviewees, most reasoning is based on naïve intuitions instead of physical principles. In fact, when asked to check if their answers were consistent with physical laws after the interview, most interviewees were able to realize that their intuition led them to the incorrect answer. When prompted after the interview to check if their answers were consistent with physical laws, all the interviewees were able to recognize that their initial responses were incorrect. This demonstrates mixed mental models with students not necessarily explicitly aware of connections unless prompted. As sometimes with the appropriate prompting, some were able to know the correct answer, this may indicate a stronger understanding of the underlying concepts, but weak contextual understanding. And for those who were not able to realize what the correct answers, they are likely to have a weaker understanding of the underlying concepts from the start.

One possible reason for graduate students having these difficulties is that there is some disconnection between the advance mathematical tools that were introduced in the advance courses and the basic conceptual understanding of the physical scenarios. This could be remedied by enforcing the students to review the advanced theory in the context of physical situations, such that they can integrate the mathematical formulation with the physical phenomenon related to conductors and insulators.

There are a few limitations to this work: the number of interviewees is low due to the time constraint of the project, which can be improved. If more first-year physics graduate students were selected to participate in this project, it would be interesting to choose those who have teaching experience related to these topics (such as calculus-based introductory E\&M), as none of the current interviewees do, and make comparison.

Acknowledgments: The author wishes to thank the physics graduate students who participated in this study.

\section{Appendix A: Test Questions}

1. Please describe the fundamental properties of conductors and insulators.

2. Now consider the following two scenarios:

(1) A hollow metal sphere is electrically neutral (no excess charge). A small amount of negative charge is suddenly placed at one point $\mathrm{P}$ on this metal sphere. If we check on this excess negative charge a few seconds later, we will find which one of the following possibilities:

(a) All of the excess charge remains right around $P$.

(b) The negative charge is evenly distributed over the outside of the sphere, while the inside of the sphere is electrically neutral.

(c) Negative charge is evenly distributed both over the inside and outside of the sphere. 
(d) Negative charge is evenly distributed over the outside of the sphere, while positive charge is induced over the inside of the sphere.

(e) Most of the charge is still at point $\mathrm{P}$, but some will have spread over the sphere.

(f) There will be no excess charge left.

(2) A hollow sphere made out of electrically insulating material is electrically neutral. A small amount of negative charge is suddenly placed at one point $P$ on this sphere. If we check on this excess negative charge a few seconds later, we will find which one of the following possibilities:

(a) All of the excess charge remains right around $P$.

(b) The negative charge is evenly distributed over the outside of the sphere, while the inside of the sphere is electrically neutral.

(c) Negative charge is evenly distributed both over the inside and outside of the sphere.

(d) Negative charge is evenly distributed over the outside of the sphere, while positive charge is induced over the inside of the sphere.

(e) Most of the charge is still at point $\mathrm{P}$, but some will have spread over the sphere.

(f) There will be no excess charge left.

3. Now consider the following two scenarios:

(1) A thick metal spherical shell is electrically neutral (no excess charge). A point charge with $+q$ is placed at the center of the spherical shell. Please draw the charge distribution everywhere on the spherical shell.

(A) Please draw the charge distribution everywhere on the spherical shell.

(B) Is the electric field the same at point $\mathrm{A}, \mathrm{B}$, and C?

(C) Please draw the electric field lines in all three regions.

(2) A thick metal spherical shell is electrically neutral. A point charge with $+q$ is placed off-centered within the spherical shell.

(A) Please draw the charge distribution everywhere on the spherical shell.

(B) Is the electric field the same at point A, B, and C?

(C) Please draw the electric field lines in all three regions.

- A

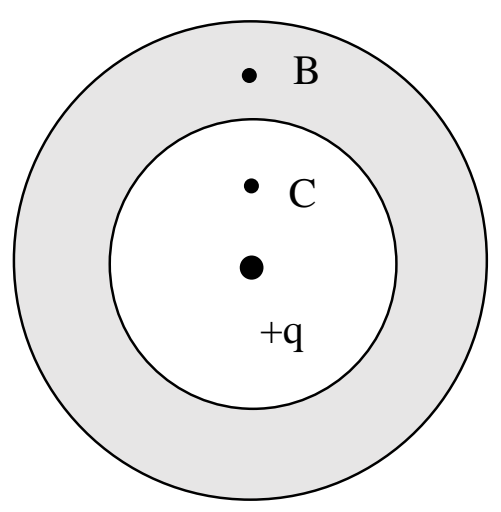

- $\mathrm{A}$

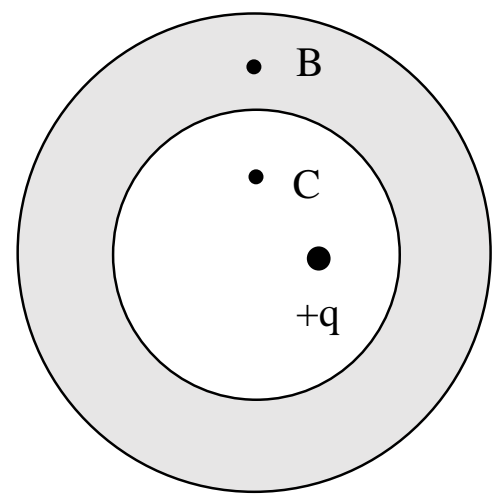




\section{References:}

Chi, M. T. (1994). Chapter 1. In The Thinking Aloud Method. London: Academic Press.

Demirci, N. (2005). University Students' Conceptual Difficulties about Electricity and Magnetism Concepts. 23th International Physics Congress, 13-16.

Hazelton, R. L., Stetzer, M. R., Heron, P. R., \& Shaffer, P. S. (2013). Investigating student ability to apply basic electrostatics concepts to conductors. AIP Conference Proceedings. 1513, pp. 166-169. AIP.

Maloney, D. P., O'Kuma, T. L., Hieggelke, C. J., \& Van Heuvelen, A. (2001). Surveying students' conceptual knowledge of electricity and magnetism. American Journal of Physics, 69(S1), S12-S23.

Pepper, R. E., Chasteen, S. V., Pollock, S. J., \& Perkins, K. K. (2010). Our best juniors still struggle with Gauss's Law: Characterizing their difficulties. AIP Conference Proceedings. 1289, pp. 245-248. AIP.

Planinic, M. (2006). Assessment of difficulties of some conceptual areas from electricity and magnetism using the Conceptual Survey of Electricity and Magnetism. American Journal of Physics, 74(12), 1143-1148. 